\title{
Radar Antenna Selection for Direction-of-Arrival Estimations
}

\author{
Arda Atalik*†, Mustafa Yilmaz*, Orhan Arikan* \\ *Department of Electrical-Electronics Engineering \\ Bilkent University Ankara, TR-06800, Turkey \\ ${ }^{\dagger}$ Huawei R\&D Center Istanbul, TR-34768, Turkey
}

\begin{abstract}
Multi-antenna radars exhibit positively correlated detection performance with the number of elements utilized. The feasibility of refining antenna arrays to reduce cost of operation with only marginal loss of performance has attracted significant attention as utilizing a large number of elements may be prohibitively costly in terms of computation and power. Under cognitive radar paradigm, the goal is to choose an optimal or near optimal subset of elements from an antenna array of pre-specified geometry while meeting certain performance and cost criteria. In this work, we present optimization based selection methods for certain array geometries to select the best $K$-element sub-array in terms of Cramér-Rao lower bound (CRB) on direction-ofarrival (DoA) estimations. Our results indicate that it is possible to reduce $K$ up to a certain point without significant reduction in DoA estimation performance. The maximum possible reduction in $K$ depends on the operating signal-to-noise ratio (SNR) and how much performance loss is tolerated. Thus, once the operating SNR is known, it is possible to utilize fewer array elements with slight decrease in performance.
\end{abstract}

Index Terms-Antenna selection, cognitive radar, direction-ofarrival estimations

\section{INTRODUCTION}

Cognitive radar was proposed to enable radar systems to adapt the transmitter and the receiver to various target and environment scenarios based on information extracted from signal returns [1]. In this paper, we investigate methods for selecting a subset of antenna array that is optimal in terms minimizing the Cramér-Rao lower bound on DoA estimation.

MIMO systems utilize a large number of array elements to enhance bit error rates, detection performance or DoA estimation, depending on application scenarios. However, using a large number of elements incurs high operation cost in terms of computation and power consumption. Therefore, it is of interest to design methods that reduce the operation cost while retaining relevant performance metrics as much as possible. Cognitive radar [2] employs target returns to choose a subset of the antenna array to reduce the number of elements that are operational at any given time, thereby reducing operation cost without significantly degrading the system performance.

Recent approaches to this problem focused on such techniques as enumerating sub-arrays to determine one for which a lower bound on DoA estimation is minimum [3] and greedily searching sub-arrays that maximize the mutual information between measurements and the antenna pattern [4]. Another work formulates the selection problem as an optimization problem with respect to spatial correlation coefficient [5]. A more recent machine-learning based approach is proposed in [6] where the antenna selection problem is formulated as a classification problem and neural networks are used to perform antenna selection.

In this work, we investigate an optimization based method that selects the optimal K-element subset of the array with respect to Cramér-Rao lower bound on DoA estimation. The general formulation is specialized to various regular array geometries, including uniform linear arrays (ULA), uniform circular arrays (UCA), and uniform rectangular arrays (URA) as optimization problems. The optimization problems do not have closed form solutions except for certain special array geometries such as ULA. For UCA and URA, we propose numerical methods partitioning the space of possible DoA's so that the partition divides the space into subsets corresponding to the same array elements. Simulations are carried out to compare the DoA estimation performance of the selected optimal sub-array to the full array and an ensemble of randomly chosen sub-arrays of the same size. The effect of the number of sub-array elements, $\mathrm{K}$, on the DoA estimation performance is also investigated at various SNR levels.

The layout of the remainder of the paper is as follows. The next section describes the modelling of the system. In Section 3, we formulate the CRB and Fisher information for the ULA, UCA, and URA geometries and the corresponding optimization problems related to the fixed-size sub-array selection. In Section 4 we compare the mean-squared error (MSE) of the selected optimal sub-arrays with the entire arrays and average performance of randomly picked sub-arrays of the same size through simulations over a range of SNR values. The results indicate that optimal sub-array performance is close to the full array's performance and outperforms average and best performance of randomly chosen sub-arrays. Our simulations also verify that a relatively small sub-array can achieve DoA estimation performance close to the full array especially at high SNR levels.

\section{Preliminaries And Data Model}

We consider a radar system with $M$ elements in which each element illuminates a single target with a waveform $s(t)$. Assume that the received and matched filtered, demodulated signals are narrow-band and planar. Let $\mathbf{a}(\phi, \theta)$ denote the array steering vector

$$
\mathbf{a}(\phi, \theta)=\left[a_{1}(\phi, \theta), a_{2}(\phi, \theta), \ldots, a_{M}(\phi, \theta)\right]^{T}
$$


where $\phi$ is the azimuth angle and $\theta$ is the elevation angle of the source. The radar collects $M$ signals in each $N$ snapshots. The received signal for the $t^{t h}$ snapshot is [6]

$$
\mathbf{y}_{t}=\mathbf{a}(\phi, \theta) s_{t}+\mathbf{n}_{t} \quad t \in\{1,2, \ldots, N\}
$$

where $s_{t} \sim \mathcal{C N}\left(0, \sigma_{s}^{2}\right)$ and $\mathbf{n}_{t} \sim \mathcal{C N}\left(0, \sigma_{n}^{2} I_{M}\right)$ are mutually uncorrelated. In matrix form, the received signal can be written as

$$
\mathbf{Y}=\mathbf{a}(\phi, \theta) \mathbf{s}^{T}+\mathbf{N}
$$

where $\mathbf{Y}=\left[\mathbf{y}_{1}, \mathbf{y}_{2}, \ldots, \mathbf{y}_{N}\right]^{T}, \mathbf{s}=\left[s_{1}, s_{2}, \ldots, s_{N}\right]^{T}$ and $\mathbf{N}=\left[\mathbf{n}_{1}, \mathbf{n}_{2}, \ldots, \mathbf{n}_{N}\right]^{T}$. Observing $\mathbf{Y}$, the receiver estimates the direction of arrival $(\phi, \theta)$ using (approximate) maximum likelihood methods.

Our aim is to select $K<M$ elements of the radar with minimum performance reduction. Since the maximum likelihood estimators are asymptotically efficient, we choose the $K$-element sub-array which minimizes the Cramér-Rao lower bound. Thus, we minimize the asymptotic variance of the ML estimator of direction-of-arrival. The CRB of the angular estimates is given in [7]:

$$
\begin{aligned}
\operatorname{CRB}(\theta) & =\chi \frac{\frac{\partial \mathbf{a}^{H}}{\partial \phi} \frac{\partial \mathbf{a}}{\partial \phi}}{\left(\frac{\partial \mathbf{a}^{H}}{\partial \phi} \frac{\partial \mathbf{a}}{\partial \phi}\right)\left(\frac{\partial \mathbf{a}^{H}}{\partial \theta} \frac{\partial \mathbf{a}}{\partial \theta}\right)-\left(\frac{\partial \mathbf{a}^{H}}{\partial \phi} \frac{\partial \mathbf{a}}{\partial \theta}\right)^{2}} \\
\operatorname{CRB}(\phi) & =\chi \frac{\frac{\partial \mathbf{a}^{H}}{\partial \theta} \frac{\partial \mathbf{a}}{\partial \theta}}{\left(\frac{\partial \mathbf{a}^{H}}{\partial \phi} \frac{\partial \mathbf{a}}{\partial \phi}\right)\left(\frac{\partial \mathbf{a}^{H}}{\partial \theta} \frac{\partial \mathbf{a}}{\partial \theta}\right)-\left(\frac{\partial \mathbf{a}^{H}}{\partial \phi} \frac{\partial \mathbf{a}}{\partial \theta}\right)^{2}}
\end{aligned}
$$

where $\chi=\frac{1+M \sigma_{s}^{2} / \sigma_{n}^{2}}{2 N\left(1+M \sigma_{s}^{2} / \sigma_{n}^{2}\right)^{2}}$ is a function of array signalto-noise ratio and number of snapshots.

For joint estimation of $(\theta, \phi)$, one needs to find a scalar measure to optimize. There are many measures in literature such as average-variance, largest eigenvalue, and determinant criterion [8]. We prefer to use average variance criterion ( $A$ optimality) for its simplicity, i.e, we select the sub-array which minimizes the sum of CRBs.

\section{CRB Minimization For Different GeOMEtRies}

\section{A. Uniform Linear Array}

There are $M$ elements located on the z-axis with uniform spacing equal to the half wavelength. Assume that the centroid of the array is the phase reference, i.e. $z_{c}=\frac{1}{M} \sum_{i=0}^{M-1} z_{i}$. Then the manifold vector is

$$
\mathbf{a}(\theta)=\left[e^{j \frac{2 \pi}{\lambda}\left(z_{0}-z_{c}\right) \cos \theta}, \ldots, e^{j \frac{2 \pi}{\lambda}\left(z_{M-1}-z_{c}\right) \cos \theta}\right]^{T}
$$

where $z_{i}=b+i \frac{\lambda}{2}$ and $b$ is arbitrary.

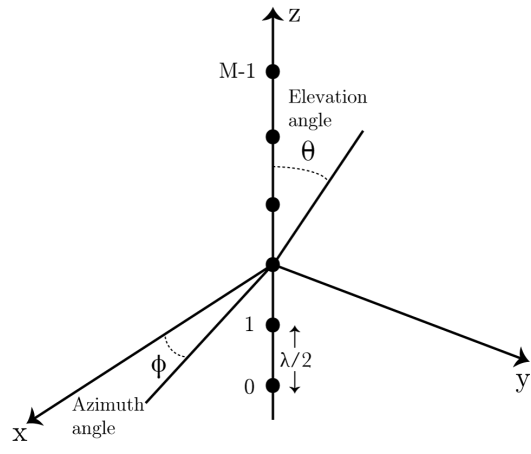

Fig. 1. Uniform Linear Array [9]

Our aim is to select $K$ elements which minimizes the CRB of $\theta$. A similar problem was analyzed in [10] and the CRB as a function of the selection vector was given as

$$
\operatorname{CRB}(\mathbf{w}, \theta)=\frac{K \csc (\theta)^{2}}{\alpha} \frac{1}{\mathbf{w}^{T} \mathbf{D} \mathbf{w}}
$$

where $\mathbf{w} \in\{0,1\}^{M}$ denotes the selection vector, $[\mathbf{D}]_{i, j}=$ $(i-j)^{2}$ with row and column indices $i, j$ and $\alpha$ is a positive function depending only on array signal-to-noise ratio and number of snapshots. Hence, the optimization problem can be written as

$$
\begin{aligned}
& \widehat{\mathbf{w}}=\underset{\mathbf{w}}{\arg \max } \mathbf{w}^{T} \mathbf{D} \mathbf{w} \\
& \text { s.t. } \mathbf{1}^{T} \mathbf{w}=K \\
& {[\mathbf{w}]_{i} \in\{0,1\} \quad \forall i \in\{1,2, \ldots, M\} }
\end{aligned}
$$

The optimization problem in (8) is non-convex due to (10) and definition of $\mathbf{D}^{1}$. Nonetheless, closed form solution exists. By definition of $\mathbf{D}, \mathbf{w}^{T} \mathbf{D} \mathbf{w}=\sum_{i, j}[\mathbf{D}]_{i, j}[\mathbf{w}]_{i}[\mathbf{w}]_{j}$ subject to $\|\mathbf{w}\|_{0}=K$ is maximized when $\mathbf{w}$ has ones in its first $\frac{K}{2}$ and last $\frac{K}{2}$ indices, i.e., we select $K$ elements from the boundary of the array.

\section{B. Uniform Circular Array}

There are $M$ elements located on a circle in the $x y$-plane. The radius of the circle is chosen such that the arc length between adjacent elements is equal to the half wavelength, i.e. $R=\frac{\lambda M}{4 \pi}$. We number the array elements starting from $\phi=0$, i.e. the azimuth angle of element $k$ is $\frac{2 \pi k}{M}$ where $k \in$ $\{0,1, \ldots, M-1\}$. For these conventions, the array manifold vector is

$$
\mathbf{a}(\phi, \theta)=\left[e^{j \frac{M}{2} \sin \theta \cos (\phi)}, \ldots, e^{j \frac{M}{2} \sin \theta \cos \left(\phi-\frac{2 \pi(M-1)}{M}\right)}\right]_{(11)}^{T}
$$

${ }^{1} \mathbf{D}$ is an indefinite matrix 


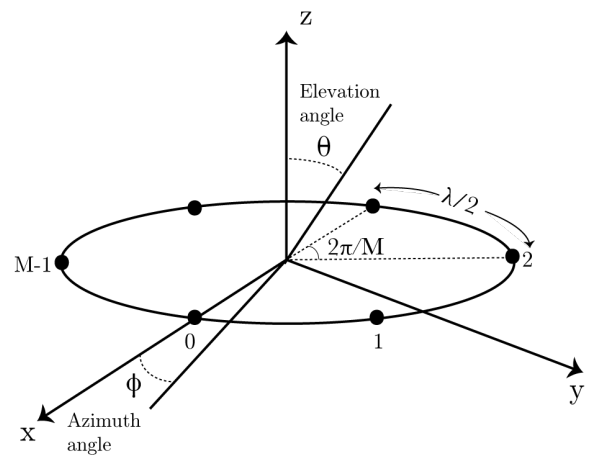

Fig. 2. Uniform Circular Array [11]

1) 1-Dimensional Case: In this scenario, the target is placed in the $x y$-plane, i.e. $\theta=90^{\circ}$ and we are interested in choosing $K$ array elements minimizing the CRB of $\phi$ or equivalently, maximizing the Fisher Information of $\phi$ as a function of the selection vector. We can calculate the Fisher Information using the equations in [7]:

$$
\begin{aligned}
F_{\phi \phi}(\mathbf{w}, \phi) & =\beta \sum_{i=0}^{M-1} \sin ^{2}\left(\phi-\frac{2 \pi i}{M}\right)[\mathbf{w}]_{i} \\
& =\frac{K \beta}{2}-\frac{\beta}{2} \sum_{i=0}^{M-1} \cos \left(2\left(\phi-\frac{2 \pi i}{M}\right)\right)[\mathbf{w}]_{i}
\end{aligned}
$$

where $K$ is the number of selected array elements, and $\beta$ is a positive function depending only on array signal-to-noise ratio and number of snapshots. Hence, the optimization problem is

$$
\begin{aligned}
& \widehat{\mathbf{w}}=\underset{\mathbf{w}}{\arg \min } \mathbf{x}^{T} \mathbf{w} \\
& \text { s.t. } \mathbf{1}^{T} \mathbf{w}=K \\
& {[\mathbf{w}]_{i} \in\{0,1\} \quad \forall i \in\{1,2, \ldots, M\} }
\end{aligned}
$$

where $[\mathbf{x}]_{i}=\cos \left(2\left(\phi-\frac{2 \pi(i-1)}{M}\right)\right) \quad i \in\{1,2, \ldots, M\}$ There is no closed form solution since the optimal selection vector depends on the azimuth angle $\phi$, i.e., given $\phi$, the optimal decision rule chooses $K$ smallest indices of $\mathbf{x}$.

Instead of directly estimating $\phi$, our aim is to partition the interval $[0,2 \pi)$ into sub-intervals $\mathcal{P}_{1}, \mathcal{P}_{2}, \ldots, \mathcal{P}_{L}$ such that the optimal selection vector is the same $\forall \phi \in \mathcal{P}_{i}$. So, we need to perform at most $L$ detections to choose the optimal sub-array.

One can find the optimal partitions and corresponding subarrays numerically for any $M$ by sorting $\mathrm{x}$ in ascending order for finely quantized $\phi \in[0,2 \pi)$. Our simulations suggest that for even and odd $M$, we can partition $[0,2 \pi)$ into $2 M$ and $4 M$ equal length sub-intervals respectively. As an example, let $M=5$. The sub-intervals are calculated as $\mathcal{P}_{i}=\left[\frac{\pi(i-1)}{10}, \frac{\pi i}{10}\right)$ for $i \in\{1,2, \ldots, 20\}$.

2) 2-Dimensional Case: In this case, the target is not in the $x y$-plane, and our aim is to choose $K$ elements minimizing the trace of the CRB matrix as a function of the selection vector. Using (4) and (5), we obtain

$$
\begin{aligned}
& \operatorname{CRB}(\mathbf{w}, \phi)+\operatorname{CRB}(\mathbf{w}, \theta)= \\
& \frac{\gamma \sum_{n=0}^{M-1}\left(f_{1}^{2}+f_{2}^{2}\right) w_{n}}{\left(\sum_{n=0}^{M-1} f_{1}^{2} w_{n}\right)\left(\sum_{n=0}^{M-1} f_{2}^{2} w_{n}\right)-\left(\sum_{n=0}^{M-1} f_{1} f_{2} w_{n}\right)^{2}}
\end{aligned}
$$

where $f_{1}(\phi, \theta, n)=\cos \theta \cos \left(\frac{2 n \pi}{M}-\phi\right)$

$f_{2}(\phi, \theta, n)=\sin \theta \sin \left(\frac{2 n \pi}{M}-\phi\right)$ and $\gamma$ is a positive function depending only on array signal-to-noise ratio and number of snapshots.

Using trigonometric identities, the optimization problem for minimizing (17) can be written in a more compact form

$$
\begin{aligned}
& \widehat{\mathbf{w}}=\underset{\mathbf{w}}{\arg \min } \frac{2 K+\mathbf{a}^{T} \mathbf{w}}{K^{2}+\mathbf{w}^{T} \mathbf{A} \mathbf{w}} \\
& \text { s.t. } \mathbf{1}^{T} \mathbf{w}=K \\
& \quad[\mathbf{w}]_{i} \in\{0,1\} \quad \forall i \in\{1,2, \ldots, M\}
\end{aligned}
$$

where

$$
\begin{aligned}
{[\mathbf{a}]_{i}=} & \cos \left(\frac{4(i-1) \pi}{M}-2(\phi+\theta)\right) \\
& +\cos \left(\frac{4(i-1) \pi}{M}-2(\phi-\theta)\right) \quad \forall i \in\{1,2, \ldots, M\}
\end{aligned}
$$

$$
\begin{aligned}
{[\mathbf{A}]_{i j}=} & \cos \left(\frac{4(i-1) \pi}{M}-2 \phi\right)-\cos \left(\frac{4(j-1) \pi}{M}-2 \phi\right) \\
& -\cos \left(\frac{4(j-i) \pi}{M}\right) \quad \forall i, j \in\{1,2, \ldots, M\}
\end{aligned}
$$

Due to the objective function and $\{0,1\}$ constraints, (18) is not convex. Although the previous problems (8) and (14) are also non-convex, given $\phi$ and $\theta$, we do not have to check all $\left(\begin{array}{c}M \\ K\end{array}\right)$ combinations to find the optimal sub-array. On the contrary, in (18), it is necessary to check all $\left(\begin{array}{l}M \\ K\end{array}\right)$ sub-arrays to find the optimal one. For large $M$, this task is tedious and impractical. One solution to this problem is relaxation of the $[\mathbf{w}]_{i} \in\{0,1\}$ constraint into $[\mathbf{w}]_{i} \in[0,1]$ :

$$
\begin{aligned}
& \widehat{\mathbf{w}}=\underset{\mathbf{w}}{\arg \min } \frac{2 K+\mathbf{a}^{T} \mathbf{w}}{K^{2}+\mathbf{w}^{T} \mathbf{A} \mathbf{w}} \\
& \text { s.t. } \mathbf{1}^{T} \mathbf{w}=K \\
& \quad[\mathbf{w}]_{i} \in[0,1] \quad \forall i \in\{1,2, \ldots, M\}
\end{aligned}
$$

One can solve (23) using a constrained nonlinear optimization algorithm such as Interior-Point [12] [13]. After solving the optimization, we can select the $K$ largest elements of $\widehat{\mathbf{w}}$ to construct the selection vector. However, we can possibly make some improvement by a local optimization method described in [14]: we can order $\widehat{\mathbf{w}}$ and check for possible swapping of $K$ selected elements (sorted in ascending order) and $M-K$ unselected elements (sorted in descending order).

For any $M$ and $K$, we can solve the relaxed problem and perform local optimization for sufficiently many $\phi \in \Phi$ and 
$\theta \in \Theta$ to obtain the partitions of $\Phi, \Theta$. Thus, we can find the optimal selection vector by performing detections in azimuth and elevation cells as in the 1-dimensional case.

\section{Uniform Rectangular Array}

There are $M=M_{x} M_{y}$ elements, laid out as a uniform rectangular grid in the $x y$-plane, where $M_{x}$ and $M_{y}$ denote the number of elements along the $x$ and $y$ axis, respectively. The spacing between adjacent array elements in either direction is chosen to be equal to the half wavelength, $\lambda / 2$.

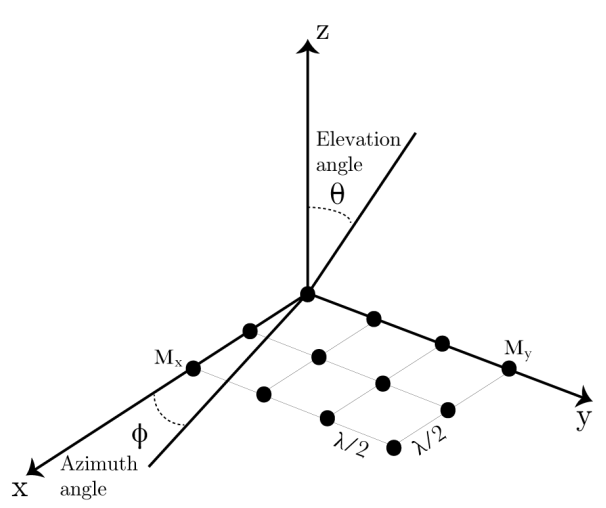

Fig. 3. Uniform Rectangular Array [15]

For the URA, the array element $\left(m_{x}, m_{y}\right)$ is located at $\left(\left(m_{x}-1\right) d_{x},\left(m_{y}-1\right) d_{y}, 0\right) \quad \forall m_{x} \in\left\{1,2, \ldots, M_{x}\right\}$ and $\forall m_{y} \in\left\{1,2, \ldots, M_{y}\right\}$, which leads to the following expression for the array steering vector [15]

$$
\mathbf{a}\left(\psi_{x}, \psi_{y}\right)=\mathbf{a}_{y}\left(\psi_{y}\right) \otimes \mathbf{a}_{x}\left(\psi_{x}\right)
$$

where $\otimes$ represents the Kronecker product and the vectors $\mathbf{a}_{x}\left(\psi_{x}\right)$ and $\mathbf{a}_{y}\left(\psi_{y}\right)$ given by

$$
\begin{aligned}
& {\left[\mathbf{a}_{x}\left(\psi_{x}\right)\right]_{m_{x}}=e^{j \psi_{x}\left(m_{x}-1\right)} \forall m_{x} \in\left\{1,2, \ldots, M_{x}\right\}} \\
& {\left[\mathbf{a}_{y}\left(\psi_{y}\right)\right]_{m_{y}}=e^{j \psi_{x}\left(m_{y}-1\right)} \forall m_{y} \in\left\{1,2, \ldots, M_{y}\right\}}
\end{aligned}
$$

represent the phase lag that can be attributed to deviation along the $x$ and $y$ axis, respectively and $\psi_{x}$ and $\psi_{y}$ are defined in terms of the wavenumber $\kappa=2 \pi / \lambda$ and array dimension $d_{x}$ and $d_{y}$ by

$$
\begin{aligned}
& \psi_{x}=\kappa d_{x} \sin (\theta) \cos (\phi) \\
& \psi_{y}=\kappa d_{y} \sin (\theta) \sin (\phi)
\end{aligned}
$$

Taking inter-element spacing in either direction to be half the wavelength, the relations (29) and (30) can be reformulated as

$$
\begin{aligned}
& \psi_{x}=\pi \sin (\theta) \cos (\phi) \\
& \psi_{y}=\pi \sin (\theta) \sin (\phi)
\end{aligned}
$$

1) 1-Dimensional Case: Similar to the UCA, we first consider the case where the source is collocated with the antenna array on the same plane. That is, $\theta=90^{\circ}$. The Fisher Information for estimating the azimuth angle $\phi$ can be calculated by taking inner products of the derivatives of the steering vector to yield [7]:

$$
\begin{aligned}
F_{\phi \phi}(\mathbf{w}, \phi)=\eta \sum_{m_{x}=1}^{M_{x}} \sum_{m_{y}=1}^{M_{y}}\left(-\left(m_{x}-1\right) \sin (\phi)+\right. \\
\left.\left(m_{y}-1\right) \cos (\phi)\right)^{2}[\mathbf{w}]_{m_{x}, m_{y}}
\end{aligned}
$$

where $\eta$ is a positive function depending only on array signal-to-noise ratio and number of snapshots. The resulting optimization problem is

$$
\begin{aligned}
& \widehat{\mathbf{w}}=\underset{\mathbf{w}}{\arg \max } \mathbf{x}^{T} \mathbf{w} \\
& \text { s.t. } \mathbf{1}^{T} \mathbf{w}=K \\
& \quad[\mathbf{w}]_{i} \in\{0,1\} \quad \forall i \in\{1,2, \ldots, M\}
\end{aligned}
$$

where, $[\mathbf{x}]_{m_{x}+M_{x}\left(m_{y}-1\right)}=\left(-\left(m_{x}-1\right) \sin (\phi)+\left(m_{y}-\right.\right.$ 1) $\cos (\theta))^{2}$.

As in the case of UCA, there is no closed form solution to (34) and the same partitioning strategy is employed. We find partitions of the interval $[0,2 \pi)$ into sub-intervals $\mathcal{P}_{1}, \mathcal{P}_{2}, \ldots, \mathcal{P}_{L}$ such that the optimal selection vector is the same $\forall \phi \in \mathcal{P}_{i}$. The optimal partitions are found numerically as in UCA. The array geometry does not permit partitions as uniformly as UCA. Nonetheless, they are also intervals, with boundaries determined by array size and inter-element spacing.

2) 2-Dimensional Case: The trace of the CRB matrix can be formulated as

$$
\operatorname{CRB}(\mathbf{w}, \phi)+\operatorname{CRB}(\mathbf{w}, \theta)=\frac{\zeta(X+Y)}{X Y-Z^{2}}
$$

where

$$
\begin{aligned}
& X=\sum_{m_{x}=1}^{M_{x}} \sum_{m_{y}=1}^{M_{y}} f_{1}^{2} w_{m_{x}, m_{y}} \\
& Y=\sum_{m_{x}=1}^{M_{x}} \sum_{m_{y}=1}^{M_{y}} f_{2}^{2} w_{m_{x}, m_{y}} \\
& Z=\sum_{m_{x}=1}^{M_{x}} \sum_{m_{y}=1}^{M_{y}} f_{1} f_{2} w_{m_{x}, m_{y}}
\end{aligned}
$$

$$
\begin{aligned}
f_{1}\left(\phi, \theta, m_{x}, m_{y}\right)= & -\left(m_{x}-1\right) \sin (\theta) \sin (\phi) \\
& +\left(m_{y}-1\right) \sin (\theta) \cos (\phi) \\
f_{2}\left(\phi, \theta, m_{x}, m_{y}\right)= & \left(m_{x}-1\right) \cos (\theta) \cos (\phi) \\
& +\left(m_{y}-1\right) \cos (\theta) \sin (\phi)
\end{aligned}
$$

and $\zeta$ is a positive function depending only on array signalto-noise ratio and number of snapshots.

This leads to the optimization problem

$$
\begin{aligned}
& \widehat{\mathbf{w}}=\underset{\mathbf{w}}{\arg \min } \frac{X+Y}{X Y-Z^{2}} \\
& \text { s.t. } \mathbf{1}^{T} \mathbf{w}=K \\
& \quad[\mathbf{w}]_{i} \in\{0,1\} \quad \forall i \in\{1,2, \ldots, M\}
\end{aligned}
$$




\section{Simulation Results}

In this section, we provide the estimation performance of our proposed method. We used Beamscan spatial sprectrum estimator which utilizes a narrowband conventional beamformer and compared the performance of full-array, random $K$-subarray, and CRB minimizer $K$-sub-array. As a performance metric, we chose mean square estimation error (MSE) in degrees. In all simulations, we used $N=100$ snapshots.

\section{A. Uniform Linear Array}

For this part, we analyzed a ULA with $M=12$ elements. We consider $K=6$ and $K=8$ element sub-arrays. Since $M$ is small, we ran the simulations with all possible sub-arrays.
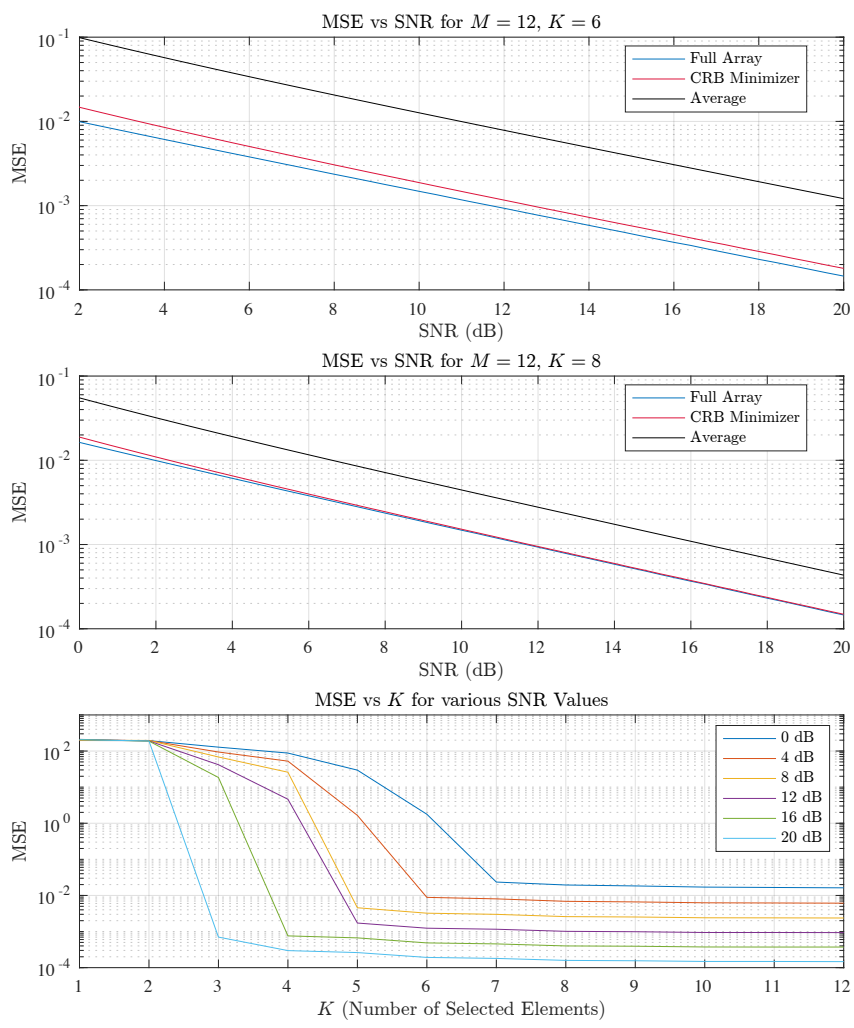

Fig. 4. Azimuth Estimation Performance for ULA

We observed that CRB minimizer sub-array has the lowest mean square error amongst all possible sub-arrays, and it achieves a performance similar to the full array.

For $K=6$, an MSE of $10^{-2}$ is achieved at $2 \mathrm{~dB}, 3.2 \mathrm{~dB}$ and $11 \mathrm{~dB}$ respectively for the full array, $\mathrm{CRB}$ minimizer subarray, and random sub-array. For $K=8$, the same MSE is achieved at $2.8 \mathrm{~dB}$ and $6.8 \mathrm{~dB}$ for the $\mathrm{CRB}$ minimizer and random sub-array. The bottom figure indicates that MSE vs $K$ curve exhibits a knee point for each SNR, i.e., once the operating SNR is known, it is possible to use fewer number of elements with negligible performance loss.

\section{B. Uniform Circular Array}

1) 1-dimensional Case: We consider a UCA with $M=$ 30 elements. Our aim is to choose $K=18$ elements and compare the estimation performance of different sub-arrays. For this case, the target is in the $x y$-plane and its azimuth direction-of-arrival is $30^{\circ} .{ }^{23} \mathrm{We}$ would like to compare the MSE of the azimuth estimation. Since checking all $\left(\begin{array}{l}30 \\ 18\end{array}\right)$ subarrays is not practical, we randomly choose 150 sub-arrays twice and average their MSE to estimate the random sub-array performance. In Fig. 5, we plot the MSE vs SNR of the fullarray, CRB minimizer sub-array, averages of the two groups of random sub-arrays, and the random best sub-array. ${ }^{4}$ At the bottom, we also plot the MSE vs $K$.

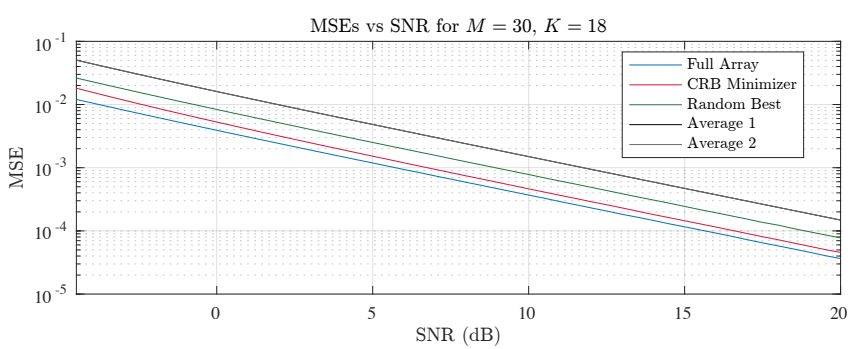

MSE vs $K$ for various SNR Values

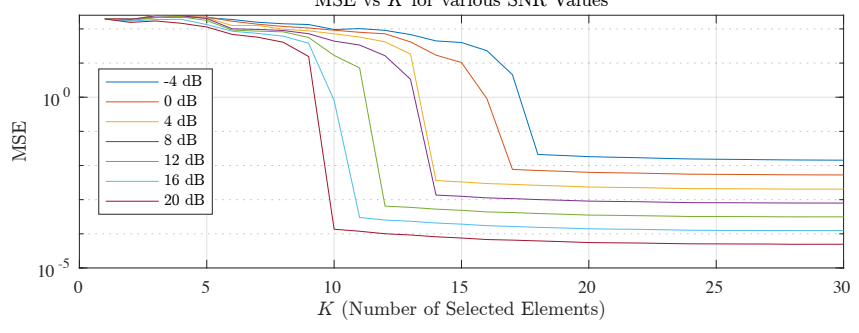

Fig. 5. Azimuth Estimation Performance for UCA

MSE of $10^{-2}$ is achieved at $-4 \mathrm{~dB},-2.5 \mathrm{~dB},-1 \mathrm{~dB}, 2$ $\mathrm{dB}$ respectively for the full array, $\mathrm{CRB}$ minimizer sub-array, random best sub-array, and random sub-array. Similar to ULA, the CRB minimizer sub-array has the lowest mean square error amongst randomly chosen 300 sub-arrays, and it achieves a performance similar to the full array. As in the ULA case, MSE vs $K$ curve exhibits a knee point for each SNR. Therefore, once the operating SNR is known it is possible to use a subset of elements with negligible performance loss.

2) 2-dimensional Case: We consider UCA with the same parameters, but now the target has a direction-of-arrival $(\phi, \theta)=\left(30^{\circ}, 75^{\circ}\right)$ and we estimate both azimuth and elevation. We would like to compare the sum of mean square errors of $\phi$ and $\theta$ for the full-array and different $K$-sub-arrays. As in the 1-dimensional case, we randomly select 15 sub-arrays twice and average their performance to estimate the random sub-array performance ${ }^{5}$.

In Fig. 6, we plot the sum of mean square estimation errors of the full-array, CRB minimizer sub-array, random subarray, and the best sub-array amongst 30 random sub-arrays.

\footnotetext{
${ }^{2}$ Our simulations suggest that the performance curves of different target azimuth angles are similar.

${ }^{3}$ Radar system has the perfect knowledge of $\theta=90^{\circ}$

4 amongst 300 random sub-arrays

${ }^{5}$ due to computational constraints, we could not select more
} 
Similar to the 1-dimensional case, the CRB minimizer subarray outperforms the best of randomly chosen sub-arrays. However, in this scenario, the performance gap between the full-array and CRB minimizer is significantly larger than that of Fig. 5: sum of MSE of $10^{-1}$ is achieved at $-0.5 \mathrm{~dB}, 1.75$ $\mathrm{dB}, 2.5 \mathrm{~dB}$, and $4 \mathrm{~dB}$ respectively for the full-array, CRB minimizer sub-array, random best sub-array ${ }^{6}$, and random subarray.

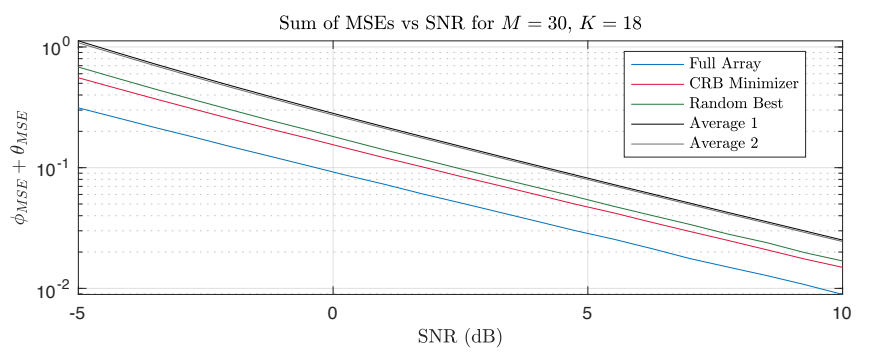

Fig. 6. Azimuth and Elevation Joint Estimation Performance for UCA

\section{Uniform Rectangular Array}

We consider a URA with $M=25$ elements in a square grid with $M_{x}=5$ and $M_{y}=5$. The target is in the $x y$ plane and its azimuth angle is $30^{\circ}$ as in UCA simulation. We choose a sub-array of $K=16$ elements and compare the resulting DoA estimation MSE with those of the full array and average of random sub-arrays. We randomly choose 50 sub-arrays twice and average their MSE to estimate random sub-array performance. In Fig. 7 we plot the MSE of the full-array, CRB minimizing sub-array, random best sub-array, and averages of two sets of random sub-arrays and the MSE corresponding to different sub-array sizes at various SNRs.
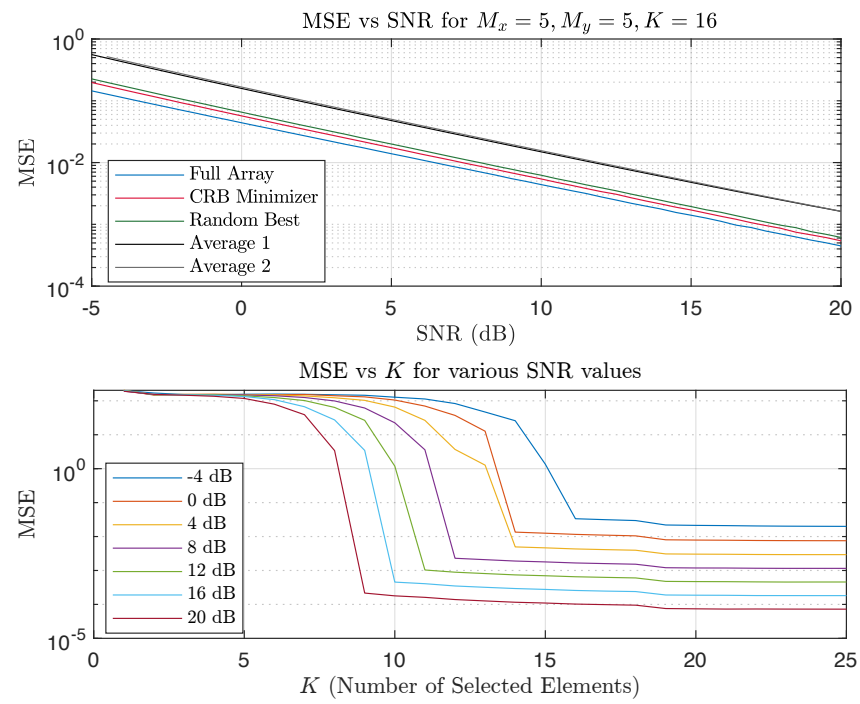

Fig. 7. Azimuth Estimation Performance for UCA

MSE of $10^{-2}$ is achieved at $6.5 \mathrm{~dB}, 7.5 \mathrm{~dB}, 8.2 \mathrm{~dB}$, and 11.7 respectively for the full array, $\mathrm{CRB}$ minimizer sub-array, the random best sub-array and averages of random sub-arrays. The performance is virtually unchanged beyond the knee point.

\section{Summary}

$K$-element antenna selection problem can be written as optimization of a desired performance metric. In general, it is a difficult combinatorial problem since the optimal solution requires checking the desired metric for all $\left(\begin{array}{c}M \\ K\end{array}\right)$ subarrays. In this work, we present a computationally simpler sub-array selection method for three commonly used array geometries. We choose the CRB minimizer sub-array. Hence, we select the sub-array which minimizes the asymptotic variance of the maximum-likelihood direction-of-arrival estimators. Simulations show that the proposed method has a decent performance: MSE of CRB minimizer sub-array is close to that of full-array and is significantly better than random selection.

\section{REFERENCES}

[1] S. Haykin, "Cognitive radar: a way of the future," IEEE Signal Processing Magazine, vol. 23, no. 1, pp. 30-40, Jan 2006.

[2] C. Baylis, M. Fellows, L. Cohen, and R. J. Marks II, "Solving the spectrum crisis: Intelligent, reconfigurable microwave transmitter amplifiers for cognitive radar,' IEEE Microwave Magazine, vol. 15, no. 5, pp. 94 107, 2014.

[3] J. Tabrikian, O. Isaacs, and I. Bilik, "Cognitive antenna selection for doa estimation in automotive radar," in 2016 IEEE Radar Conference (RadarConf), 2016, pp. 1-5.

[4] G. Shulkind, S. Jegelka, and G. W. Wornell, "Multiple wavelength sensing array design," in 2017 IEEE International Conference on Acoustics, Speech and Signal Processing (ICASSP), 2017, pp. 3424-3428.

[5] H. Nosrati, E. Aboutanios, and D. B. Smith, "Receiver-transmitter pair selection in mimo phased array radar," in 2017 IEEE International Conference on Acoustics, Speech and Signal Processing (ICASSP), 2017, pp. $3206-3210$

[6] A. M. Elbir, K. V. Mishra, and Y. C. Eldar, "Cognitive radar antenna selection via deep learning," IET Radar, Sonar \& Navigation, vol. 13, no. 6, p. 871-880, Jan 2019.

[7] C. Chambers, T. C. Tozer, K. C. Sharman, and T. S. Durrani, "Temporal and spatial sampling influence on the estimates of superimposed narrowband signals: when less can mean more," IEEE Transactions on Signal Processing, vol. 44, no. 12, pp. 3085-3098, 1996.

[8] S. P. Chepuri and G. Leus, "Sensor selection for estimation, filtering, and detection," in 2014 International Conference on Signal Processing and Communications (SPCOM), 2014, pp. 1-5.

[9] V. T. H. L., Arrays and Spatial Filters. John Wiley \& Sons, Ltd, 2002, ch. 2, pp. 17-89. [Online]. Available: https://onlinelibrary.wiley.com/doi/abs/10.1002/0471221104.ch2

[10] V. Roy, S. P. Chepuri, and G. Leus, "Sparsity-enforcing sensor selection for doa estimation," in 2013 5th IEEE International Workshop on Computational Advances in Multi-Sensor Adaptive Processing (CAMSAP), 2013, pp. 340-343.

[11] V. T. H. L., Planar Arrays and Apertures. John Wiley \& Sons, Ltd, 2002, ch. 4, pp. 231-331. [Online]. Available: https://onlinelibrary.wiley.com/doi/abs/10.1002/0471221104.ch4

[12] R. H. Byrd, M. E. Hribar, and J. Nocedal, "An interior point algorithm for large-scale nonlinear programming," SIAM J. on Optimization, vol. 9, no. 4, p. 877-900, Apr. 1999. [Online]. Available: https://doi.org/10.1137/S1052623497325107

[13] R. H. Byrd, J. C. Gilbert, and J. Nocedal, "A trust region method based on interior point techniques for nonlinear programming," Mathematical programming, vol. 89 , no. 1 , pp. 149-185, 2000.

[14] S. Joshi and S. Boyd, "Sensor selection via convex optimization," IEEE Transactions on Signal Processing, vol. 57, no. 2, pp. 451-462, 2009.

[15] P. Heidenreich, A. M. Zoubir, and M. Rubsamen, "Joint 2-d doa estimation and phase calibration for uniform rectangular arrays," Trans. Sig. Proc., vol. 60, no. 9, p. 4683-4693, Sep. 2012. [Online]. Available: https://doi.org/10.1109/TSP.2012.2203125

${ }^{6}$ amongst 30 random sub-arrays 